\title{
PROPUESTA PEDAGÓGICA EN TORNO A TÓPICOS INTRODUCTORIOS DE FÍSICA NUCLEAR
}

\section{PEDAGOGICAL APPROACH AROUND INTRODUCTORY TOPICS OF NUCLEAR PHYSICS}

\author{
E.P.Ramos G. ${ }^{1}$ \\ J.J.Peralta $\mathrm{M}^{2}$ \\ I.A.Monroy $\mathrm{C}^{3}$ \\ G.Cardona R. ${ }^{4}$
}

\begin{abstract}
Resumen
Investigaciones en el área de la enseñanza de la física en el ámbito escolar han demostrado una gran tendencia en diferentes países a proponer nuevos objetivos dentro de ésta, entre los cuales además se encuentra el reconocimiento y descripción de conceptos introductorios de física moderna en la secundaria, para nuestro caso de interés en particular la enseñanza de la física nuclear. Por otra parte, la forma como son abordadas muchas de las temáticas involucradas no suponen una metodología eficiente, lo que plantea la necesidad de un nuevo enfoque a la hora de abordar éste tipo de tópicos en la escuela, para así optimizar la enseñanza de estos. Se proponen diferentes actividades desde la metodología del aprendizaje cooperativo-colaborativo con el fin de abordar la enseñanza de temas introductorios de física nuclear (estructura atómica y fusión nuclear); en donde estos son afrontados a partir de experiencias en el aula que simulen los fenómenos que están ligados a las diferentes temáticas. Anexo a las actividades se incluyeron animaciones para respaldar el material empleado en el aula.
\end{abstract}

Palabras Clave: Aprendizaje cooperativo, física nuclear.

\section{Abstract}

Teaching physics research in school environment has shown a great tendency in different countries to propose new objectives inside it, among which are also, the recognition and description of introductory concepts about modern physics in high school, in our particular interest, teaching of nuclear physics. On the other hand, the methods used in teaching of some of those topics do not suppose an efficient methodology, raising the need of a new focus to approach this type of topics to optimize their learning in school. We propose different activities from cooperative-colaborative learning methodology, with the objective

\footnotetext{
${ }^{1}$ Universidad Pedagógica Nacional, Bogotá. dfi546_eramos@pedagogica.edu.co

${ }^{2}$ Universidad Pedagógica Nacional, Bogotá. dfi541_jperalta@pedagogica.edu.co

${ }^{3}$ Universidad Pedagógica Nacional, Bogotá. dfi541_jperalta@pedagogica.edu.co

${ }^{4}$ Universidad Pedagógica Nacional, Bogotá. gcardona@pedagogica.edu.co
} 
$5^{\circ}$ CONGRESO NACIONAL DE ENSEÑANZA DE LA FÍSICA.

Universidad Pedagógica Nacional (U. P. N)

Universidad Distrital Francisco José de Caldas (U. D. F. J. C)

Bogotá, Colombia. 16 al 20 de mayo 2011

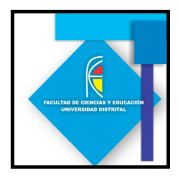

to approach the teaching of introductory topics of nuclear physics (atomic structure and nuclear fusion); where these are teach from different experiences in the classroom that simulate the phenomena that are related to the different themes. Adjunct to the activities we included animations that support the material used in class.

Keywords: Cooperative learning, nuclear physics

\section{Introducción}

Después de realizar una revisión bibliográfica en textos escolares empleados actualmente en la enseñanza de la física en Bogotá, se encuentra que existe muy poca difusión de textos que aborden tópicos introductorios de física nuclear, una temática que pocas veces se presenta en el ámbito escolar en Colombia, pero que es centro de estudio en la actualidad tanto en el ámbito de la investigación a nivel científico como educativo. Por otro lado, existen numerosas investigaciones que han mostrado las falencias que se presentan al emplear metodologías tradicionales en la enseñanza de la física en general (Arlego, M. Otero, M.R. Fanaro, M.A., 1957), lo cual crea la necesidad de pensar una metodología que permita optimizar los procesos de su enseñanza en el aula (Ferreiro, R., 2007).

Con base en esto se recopilaron actividades que utilizaran materiales de bajo costo y que permitieran, tanto representar ideas esenciales para el entendimiento de los tópicos propuestos de física nuclear: Estructura atómica y Fusión nuclear, como acoplarse a la metodología empleada durante la implementación. Las actividades que se explicitaran más en detalle posteriormente, fueron desarrolladas con una población de 30 estudiantes de la Universidad Pedagógica Nacional de primer semestre del programa académico Licenciatura en física, población que aunque no pertenece en particular a programas de educación media, sí se acerca al nivel conceptual del mismo, con el beneficio de su particular interés por la física.

\section{Metodología empleada:}

Se propone un enfoque desde el aprendizaje colaborativo enmarcado en el constructivismo, en la que todos los estudiantes se constituyen líderes en la búsqueda y construcción de conocimiento por medio de la participación activa (Calzadilla, M.E., 1986), la cual involucra la intervención tanto individual como grupal, permitiendo así una reestructuración del pensamiento en conjunto (Jones, J.L. Jones, K.A., 2008). Los diferentes puntos de vista obtenidos de los debates generados de la observación a las diferentes experiencias, permitieron reconstruir las ideas que llevaron a las conclusiones y consolidación de los conceptos planteados ligados a la física nuclear.

\section{Simulaciones y actividades en el aula: Midiendo en el mundo microscópico:}

Introducción a la actividad: En el mundo microscópico la manera en como medimos y determinamos la estructura de la materia es diferente a como lo hacemos macroscópicamente. La actividad consiste en determinar las formas de diferentes figuras geométricas de madera colocadas bajo una caja de cartón a partir de choques con canicas (figura 1). Esta actividad permite simular como las interacciones en el mundo microscópico 
$5^{\circ}$ CONGRESO NACIONAL DE ENSEÑANZA DE LA FÍSICA.

Universidad Pedagógica Nacional (U. P. N)

Universidad Distrital Francisco José de Caldas (U. D. F. J. C)

Bogotá, Colombia. 16 al 20 de mayo 2011

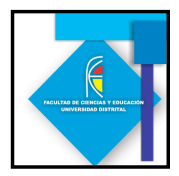

y los comportamientos que se dan gracias a éstas, nos dan una idea aproximada de la estructura de la materia (CPEP Contemporary Physics Education Project, 2010).

Actividad de aula: Se formulan diferentes preguntas para enfocar la atención de los estudiantes en torno al tema propuesto: ¿Qué es medir?, ¿Qué necesitamos para medir? A partir de estas preguntas se obtienen diferentes conclusiones de los estudiantes producto de los pequeños debates que se generaron, entre las que se resaltan: medir es observar, analizar, interpretar y mirar un comportamiento.

Se propone la actividad de aula; colocando una caja de cartón en el piso de tal manera que no se pueda ver la figura de madera que se ubica bajo ella, se plantea un nuevo interrogante, ¿Es posible medir la forma y tamaño de la figura bajo la caja sin levantarla?, con lo cual se generan discusiones en torno al método a emplear, concluyéndose así que no es posible usando ninguno de los 5 sentidos que conocen.

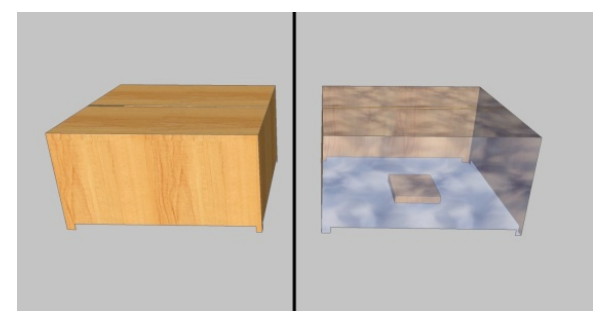

Figura 1

Se les plantea la opción de lanzar canicas bajo la caja (la caja está diseñada de tal manera que las canicas pueden pasar bajo ella sin problema) y se les reparten canicas a todos los estudiantes. Se les propone medir a partir de las colisiones entre las canicas y la figura, su tamaño y forma, dejando libre el método de medición empleado por cada uno de ellos. Se finaliza la actividad exponiendo las posibles figuras que cada estudiante plantea junto con su método de medición (algunos estudiantes observaban los ángulos de salida de las canicas para medir la figura, iotros no!). Con base en esto se hace un contraste entre los métodos usados, y se concluye en conjunto que existen métodos de medición indirecta, lo cual permite introducir cómo en el mundo microscópico medimos gracias a interacciones.

\section{Simulación del experimento de la lámina de oro:}

Introducción a la actividad: En esta experiencia se aborda el modelo atómico propuesto por Ernest Rutherford a partir de los resultados obtenidos en el experimento de la lámina de oro, en el cual partículas alfa al interactuar con la muestra eran significativamente desviadas reclamando así una nueva forma de describir la materia.

En esta actividad se presenta una simulación computacional desarrollada en java por el grupo de investigación de la Universidad de Colorado PhET development team of interactive simulations, y una animación generada en Flash para este fin desarrollada en la Universidad Pedagógica Nacional por los autores del presente documento. Con base en 
$5^{\circ}$ CONGRESO NACIONAL DE ENSEÑANZA DE LA FÍSICA.

Universidad Pedagógica Nacional (U. P. N)

Universidad Distrital Francisco José de Caldas (U. D. F. J. C)

Bogotá, Colombia. 16 al 20 de mayo 2011

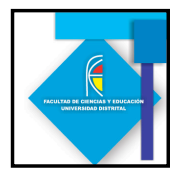

este material interactivo y en las conclusiones obtenidas en la actividad midiendo en el mundo microscópico, se reconstruyó junto a los estudiantes las premisas que llevaron a Rutherford a proponer su modelo atómico en 1909.

Actividad de aula: El comienzo de la actividad se dirige a formular preguntas en torno a la noción de átomo, núcleo y su estructura: ¿Qué es un átomo?, ¿Cómo es un átomo?, ¿Por qué cree que el átomo es así si no se puede ver?, ¿Cómo podemos saber la estructura del átomo? En la búsqueda de las respuestas a estas preguntas surgen ideas como: el átomo es una división pequeña de la materia y está constituido por un núcleo y partículas girando alrededor de éste, sin embargo se despierta la curiosidad por saber porque el átomo es visto así.

A partir de las diversas opiniones de los estudiantes, se concluye que la manera más factible para evidenciar la estructura de la materia es interactuando con ella (se hace referencia acerca de la medición indirecta en la experiencia midiendo en el mundo microscópico).

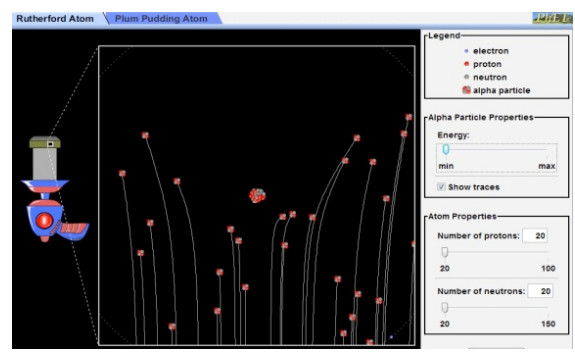

Figura 2

Continuo a las diversas opiniones e hipótesis, se les presenta la simulación desarrollada en java, en la cual se muestra como una nube de electrones no interactúa con las partículas, lo que permite proponer la pregunta ¿Cómo el experimento da cuenta de la estructura de la materia?

Seguido a esto se presenta de nuevo la simulación en java, mostrando en este caso como si existiera un núcleo atómico las partículas interactuarían con éste (figura 2), lo cual permite concluir en conjunto que el experimento en 1909 debió arrojar la segunda conclusión y no la primera. Se finalizó la experiencia mostrando la animación desarrollada en flash para articular las conclusiones de los estudiantes.

\section{Experimentando con la Fusión nuclear}

Introducción a la actividad: La Fusión nuclear es un proceso en el cual núcleos atómicos livianos reaccionan formando un núcleo más pesado. La actividad consiste en colocar tapas de gaseosa con velcro pegado a su respaldo en el interior de una caja de cartón para que al agitarla, unas tapas queden pegadas con otras (figura 3). La caja de cartón representará un reactor nuclear mientras que las tapas núcleos livianos. La manera en cómo se agite la caja representará la temperatura $T$ a la que sometemos la muestra, (asociando ésta a la velocidad de las partículas) de tal manera que si agitamos suavemente 0 bruscamente la caja se representarán bajas y altas temperaturas respectivamente. Las otras dos variables involucradas serán el tiempo de exposición $t$ y el 
$5^{\circ}$ CONGRESO NACIONAL DE ENSEÑANZA DE LA FÍSICA.

Universidad Pedagógica Nacional (U. P. N)

Universidad Distrital Francisco José de Caldas (U. D. F. J. C)

Bogotá, Colombia. 16 al 20 de mayo 2011

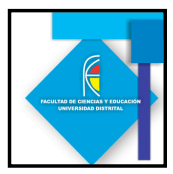

número de tapas dentro de la caja $n$, el cual tendrá relación directa con la densidad (CPEP Contemporary Physics Education Project, 2010).

Actividad en el aula:

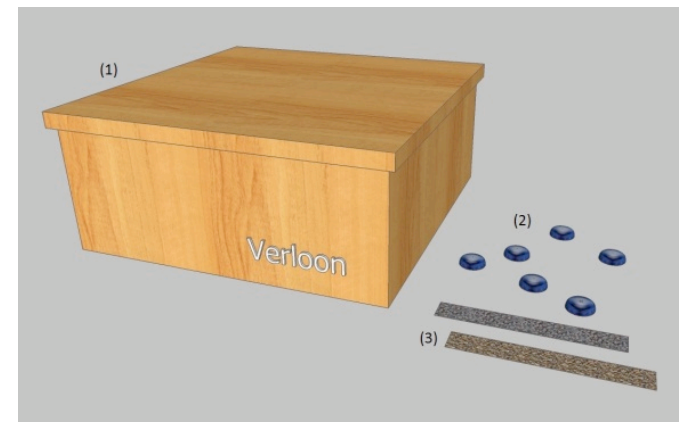

Figura 3

Al inicio de la actividad se procedió a formular una serie de preguntas que permitieran despertar curiosidad en los estudiantes y enfocarlos hacia el tema a abordar: ¿Qué entienden por fusión?, ¿Saben que es fusión nuclear?, ¿Bajo qué condiciones se puede dar la fusión nuclear? Por medio de los puntos de vista dados por los estudiantes y a partir de un debate de esas ideas previas, concluyen y construyen que; la fusión nuclear tiene lugar cuando se atraen y se pegan dos núcleos, y la condición necesaria para que se dé es que

los núcleos sean inestables, ya que una mesa está constituida por núcleos estables y por ende no presenta fusiones.

Seguido a la propuesta de los estudiantes se plantea una simulación de la fusión nuclear a partir de la caja de cartón y las tapas de gaseosa, en donde después de agitar la caja se unen un número específico de tapas generando un "núcleo más pesado". A partir de la experiencia se varían los parámetros involucrados $(T, n, t)$ sin explicitar a que magnitud física hacen referencia estos, de manera que se plantean estas tres preguntas antes de hacer las respectivas experiencias: ¿Es más fácil que se formen núcleos más pesados con 10 ó 20 tapas?, ¿Es más factible que se genere la fusión nuclear cuando agitamos la caja por 20 s o por 10s?, ¿Hay más fusiones nucleares cuando agitamos la caja rápido o lento? Se exponen las diferentes hipótesis y se continúa con las respectivas experiencias a cada pregunta, dejando constantes las dos variables que no están asociadas a cada interrogante (en la primer experiencia se dejaron constantes el tiempo $t$ y la temperatura $7)$. Inmediatamente después de contar el número de "núcleos" generados en el "reactor" se formulan de nuevo las preguntas y se debate sobre posibles cambios en las respuestas. Al realizar diferentes mediciones y socializar las ideas de cada uno de los estudiantes, se construyen con base en estas y lo observado, las condiciones necesarias para que se dé en mayor proporción la "fusión nuclear".

Se socializan los posibles factores físicos relacionados con las variables establecidas, en donde los estudiantes asociaron decenas de magnitudes físicas mostrando así opiniones diversas. A partir del debate los estudiantes concluyen tres magnitudes físicas asociadas a la experiencia: tiempo, masa y temperatura. Los docentes aclararon como la variable $n$ 
$5^{\circ}$ CONGRESO NACIONAL DE ENSEÑANZA DE LA FÍSICA.

Universidad Pedagógica Nacional (U. P. N)

Universidad Distrital Francisco José de Caldas (U. D. F. J. C)

Bogotá, Colombia. 16 al 20 de mayo 2011

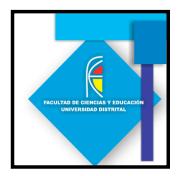

esta más asociada a la densidad de la muestra que a la masa, concluyendo en el ejemplo mencionado por los estudiantes anteriormente; la mesa no presenta fusiones nucleares ya que su masa esta confinada a un volumen grande respecto a ésta (se hace referencia a la densidad), o especificando que al realizar la experiencia con la misma caja siempre, no estábamos variando el volumen de la muestra con lo cual el cambio de la masa dentro de ese volumen constante me hacía referencia a un cambio en la densidad.

\section{Conclusiones:}

1. Al abordar temas introductorios a la física moderna se genera gran interés por parte de los estudiantes, viéndose esto evidenciado en la participación activa y compromiso de cada uno de ellos en el desarrollo de cada una de las actividades propuestas.

2. El interés mostrado por parte de los estudiantes debe ser influenciado por una de dos variables: La introducción de temáticas de física que no son comúnmente abordadas y que son parte del estudio en el mundo actual, ó la metodología empleada.

3. Gracias a la metodología empleada, en donde los estudiantes debatían constantemente sus puntos de vista a partir del desarrollo de cada una de las actividades, los estudiantes construyeron conocimientos base para la física nuclear.

\section{Referencias Bibliográficas}

Arlego, M. Otero, M.R. Fanaro, M.A. (1957). Investigación y desarrollo de propuestas didácticas para la enseñanza de la física en la escuela secundaria: Nociones cuánticas, Revista electrónica de investigación en educación en ciencias, 1, 58-74.

Calzadilla, M.E. (1986). Aprendizaje colaborativo y tecnologías de la información y la comunicación, Revista Iberoamericana de educación.

CPEP Contemporary Physics Education Project. Plasma physics and fusion, online fusion course, (10 Nov 2010). http://www.cpepweb.org/fusion.html

Ferreiro, R. (2007) Una visión de conjunto a una de las alternativas educativas más importantes en los últimos años: El aprendizaje cooperativo, revista electrónica de investigación educativa, 9 (2), 2-9.

Jones, J.L. Jones, K.A. (2008). Making cooperative learning work in the college classroom, Journal of effective teaching, 8 (2), 61-76.

PhET Interactive Science Simulations. Rutherford Scattering, (22 Feb 2011). http://phet.colorado.edu/en/simulation/rutherford-scattering

Valero, M. (2010). Física fundamental 2. Bogotá: Norma.

Lawrence Berkeley National Laboratory. Particle physics, online course, (20 Nov 2010). http://www.lbl.gov/Education/ 